Oscillation Theory for Second Order

Linear, Half-Linear, Superlinear and Sublinear Dynamic Equations 


\section{Oscillation Theory for Second Order Linear, Half-Linear, Superlinear and Sublinear Dynamic Equations}

by

Ravi P. Agarwal

Florida Institute of Technology,

Melbourne, Florida, U.S.A.

Said R. Grace

Cairo University,

Orman, Giza, Egypt

and

Donal O'Regan

National University of Ireland,

Galway, Ireland

SPRINGER-SCIENCE+BUSINESS MEDIA, B.V. 
A C.I.P. Catalogue record for this book is available from the Library of Congress.

ISBN 978-90-481-6095-2 ISBN 978-94-017-2515-6 (eBook)

DOI 10.1007/978-94-017-2515-6

Printed on acid-free paper

\section{All Rights Reserved}

(C) 2002 Springer Science+Business Media Dordrecht

Originally published by Kluwer Academic Publishers in 2002

No part of this work may be reproduced, stored in a retrieval system, or transmitted in any form or by any means, electronic, mechanical, photocopying, microfilming, recording or otherwise, without written permission from the Publisher, with the exception of any material supplied specifically for the purpose of being entered and executed on a computer system, for exclusive use by the purchaser of the work. 


\section{Contents}

Preface $\quad$ xi

\section{Chapter 1 Preliminaries}

1.0. Introduction 1

1.1. Continuability, Boundedness and Convergence to Zero 2

1.2. Some Useful Results from Analysis and Fixed Point Theorems 10

1.3. Notes and General Discussions 12

1.4. References 12

Chapter 2 Oscillation and Nonoscillation of Linear Ordinary Differential Equations

2.0. Introduction 13

2.1. Sturm Comparison Theorem 13

2.2. Nonoscillatory Characterizations and Comparison Theorems 15

2.3. Nonoscillation Criteria 25

2.4. Oscillation Criteria 35

2.5. Oscillation Criteria-Integral Averaging 40

2.6. Oscillation Criteria-Integrable Coefficients 70

2.7. Forced Oscillations $\quad 82$

2.8. Notes and General Discussions 90

2.9. References 91

Chapter 3 Oscillation and Nonoscillation of Half-Linear Differential Equations

3.0. Introduction 93

3.1. Preliminaries 94

3.2. Sturm-Type Comparison Theorems 94

3.3. Levin-Type Comparison Theorems 100

3.4. Liapunov's Inequality 105 
3.5. An Oscillation Criterion for Almost-Periodic

Sturm-Liouville Equations

3.6. On Zeros of Solutions of a Singular Half-linear Differential Equation

3.7. Nonoscillation Criteria 140

3.7.1. Nonoscillatory Characterization 140

3.7.2. Comparison Theorems 147

3.7.3. Sufficient Conditions for Nonoscillation of Equation (3.1.1) 148

3.7.4. Further Results on Nonoscillation of Equation (3.1.1) 154

3.8. Oscillation Criteria 163

3.9. Oscillation Criteria-Integral Averaging 168

3.9.1. Oscillation Theorems for Equation (3.1.1) 168

3.9.2. Interval Criteria for the Oscillation of Equation (3.1.1) 179

3.9.3. The Weighted-Average Oscillation Criteria 188

3.10. Oscillation Criteria-Integrable Coefficients 196

3.11. Oscillation of Damped and Forced Equations 205

3.11.1. Oscillation of Equation (3.11.1) 206

3.11.2. Oscillation of Equation (3.11.2) 207

3.12. Distance of Zeros of Oscillatory Solutions 210

3.13. Oscillation and Nonoscillation of Half-linear Equations with Deviating Arguments 218

3.13.1. Some Useful Lemmas 219

3.13.2. Oscillation of Equation (3.13.1) 223

3.13.3. Some Extensions and More Oscillation Criteria 232

3.13.4. Classification of Nonoscillatory Solutions 237

3.13.5. A Comparison Theorem 251

3.13.6. Oscillation of Equation (3.13.2) 253

3.13.7. Nonoscillation of Solutions of Equation (3.13.127) 259

3.14. Notes and General Discussions 267

3.15. References 268

\section{Chapter 4 Oscillation Theory for Superlinear Differential Equations}

4.0. Introduction 273

4.1. Superlinear Oscillation Criteria 273

4.2. Further Results on Superlinear Oscillations 296 
4.3. Existence of Nonoscillatory Solutions and Comparison Results 328

4.3.1. A Necessary Condition for Oscillation 328

4.3.2. Comparison of Nonlinear Equations of the Same Form 332

4.3.3. Linearization of Oscillation Theorems 339

4.4. Oscillation Criteria for Certain Nonlinear Differential Equations 341

4.5. Superlinear Forced Oscillations 358

4.6. Oscillation of Nonlinear Differential Equations with Nonlinear Damping Term 362

4.6.1. Damping with Nonlinear Growth-Oscillation Criteria 362

4.6.2. Damping with Nonlinear Growth-A Nonoscillation Theorem 367

4.7. Notes and General Discussions 371

$\begin{array}{ll}\text { 4.8. References } & 372\end{array}$

Chapter 5 Oscillation Theory for Sublinear Differential Equations

$\begin{array}{lll}\text { 5.0. Introduction } & 376\end{array}$

5.1. Sublinear Oscillation Criteria 376

5.2. Further Sublinear Oscillation Criteria 387

5.3. Linearization of Sublinear Oscillation Theorems 429

5.4. Nonoscillation Theorems for Sublinear Differential Equations 433

5.5. Oscillation Criteria for Certain Nonlinear Differential Equations 444

5.6. Notes and General Discussions 453

5.7. References 453

Chapter 6 Further Results on the Oscillation of Differential Equations

6.0. Introduction

457

6.1. Oscillation Criteria of Olech-Opial-Wazewski Type 457

6.1.1. Some Definitions and Notation 458

$\begin{array}{ll}\text { 6.1.2. Oscillation Criteria } & 459\end{array}$

6.1.3. Further Results 463

6.2. Oscillation Criteria for Half-linear Differential Equations 466

6.2.1. Preliminary Results 467

6.2.2. Oscillation Criteria 469

6.3. Oscillation Criteria via Liapunov's Second Method 476 
$\begin{array}{ll}\text { 6.3.1. Preliminaries } & 477\end{array}$

6.3.2. Oscillation Criteria 479

6.4. Notes and General Discussions 489

6.5. References 490

Chapter 7 Oscillation Results for Differential Systems

7.0. Introduction 491

7.1. Oscillation of Nonlinear Two-dimensional Differential Systems 491

7.1.1. Superlinear Case 493

7.1.2. Sublinear Case 498

7.1.3. Further Oscillation Criteria $\quad 500$

7.2. Oscillation Theorems for Linear Differential Systems 503

7.3. Oscillation Theorems for Differential Systems with Functionally Commutative Matrix Coefficients

7.4. Comparison Theorems for Operator-Valued Linear Differential Equations

7.5. Oscillation Results for Differential Systems with Forcing Terms 549

7.6. Notes and General Discussions 552

7.7. References 554

Chapter 8 Asymptotic Behavior of Solutions of Certain Differential Equations

8.0. Introduction

8.1. Asymptotic Behavior of Solutions of Nonlinear Differential Equations

8.1.1. Asymptotic Behavior of Nonoscillatory Solutions 559

8.1.2. Bounded Asymptotically Linear Solutions 562

8.1.3. Unbounded Asymptotically Linear Solutions 567

8.1.4. Further Extensions and Improvements 574

8.2. Asymptotic Behavior of Positive Solutions of Singular Emden-Fowler-Type Equations 584

8.2.1. Positive Solutions of Equation (8.2.1) 585

8.2.2. Positive Decaying Solutions of Equation (8.2.2) 595 
8.2.3. Positive Solutions of Singular Emden-Fowler-Type Systems 614

8.3. Notes and General Discussions 622

$\begin{array}{ll}\text { 8.4. References } & 624\end{array}$

\section{Chapter 9 Miscellaneous Topics}

$\begin{array}{lll}\text { 9.0. Introduction } & 625\end{array}$

9.1. Comparison and Nonoscillation Results 625

9.2. Nonoscillatory Solutions of Forced Differential Equations 632

9.3. Limit Circle Criteria and Related Properties $\begin{array}{ll}\text { for Nonlinear Equations } & 638\end{array}$

9.4. Properties of Certain Differential Equations 650

9.5. Notes and General Discussions 654

$\begin{array}{lll}\text { 9.6. } & \text { References } & 654\end{array}$

Chapter 10 Nonoscillation Theory for Multivalued Differential Equations

10.0. Introduction

$\begin{array}{ll}\text { 10.1. Preliminaries } & 656\end{array}$

$\begin{array}{ll}\text { 10.2. Differential Inclusions } & 657\end{array}$

$\begin{array}{ll}\text { 10.3. Neutral Inclusions } & 664\end{array}$

10.4. Notes and General Discussions 668

$\begin{array}{lll}\text { 10.5. References } & 668\end{array}$

Subject Index $\quad 670-672$ 


\section{Preface}

This book is devoted to the qualitative theory of second order dynamic equations. In the last 50 years the Oscillation Theory of ordinary, functional, neutral, partial, and impulsive differential equations, and their discrete versions has attracted many researchers. This has resulted in hundreds of research papers in every major mathematical journal. There are many books which deal exclusively with oscillation of solutions of differential equations. However, most of those books appeal only to researchers who already are familiar with the subject. Thus, in an effort to bring oscillation theory to a new and wider audience, in this book we present a compact, thorough, and self-contained account for second order dynamic equations. An important feature of this book is that many examples of current interest are given to illustrate the theory.

In Chapter 1, we introduce oscillation theory of second order differential equations. Here also we provide conditions which guarantee that all solutions of a particular class of second order nonlinear differential equations are continuable, bounded, and converge to zero. Our final section in this chapter states several fixed point theorems which play an important role in establishing existence criteria of nonoscillatory solutions.

It is natural to begin our discussion with second order linear differential equations. The interest in second order linear oscillations is due, in a large part, to the fact that many physical systems are modelled by such equations. We begin Chapter 2 with some of the most basic results in the theory of oscillations of linear ordinary differential equations of second order. In particular, we present Sturm and Sturm-Picone comparison theorems. Then, we provide some necessary and sufficient conditions for nonoscillation as well as some comparison theorems of Sturm type. Also we present sufficiency criteria for nonoscillation of solutions. Next, we establish sufficient conditions for oscillation of second order differential equations with alternating coefficients. Integral averaging techniques and interval criteria are two of the most important concepts in oscillation theory and both are discussed in Chapter 2. Criteria for oscillation of linear second order differential equations with integrable coefficients are also given. We conclude this chapter by discussing the problem of forced oscillations. 
In recent years the study of half-linear differential equations has become an important area of research. This is largely due to the fact that half-linear differential equations occur in a variety of real world problems such as in the study of $p$-Laplace equations, non-Newtonian fluid theory and the turbulent flow of a polytrophic gas in a porous medium. In Chapter 3, we present oscillation and nonoscillation criteria for second order half-linear differential equations. We begin with some preliminaries on half-linear differential equations, and then we present Sturm and Levin type comparison theorems and a Liapunov type inequality. Next, we provide an oscillation criterion for almost periodic Sturm-Liouville equations. In this chapter we also present a systematic study of the zeros of solutions of singular half-linear differential equations, nonoscillation characterizations (necessary and sufficient conditions), comparison results and sufficiency criteria for nonoscillation. In addition we study oscillation by employing integral and weighted averaging techniques. Here, interval criteria for the oscillation of half-linear equations will also be provided. Next, we discuss oscillation of half-linear equations with integrable coefficients, and damped and forced equations. We also derive lower bounds for the distance between consecutive zeros of an oscillatory solution. Finally in this chapter, we present a systematic study of the oscillation and nonoscillation of half-linear equations with a deviating argument. Here, classifications of nonoscillatory solutions, and existence results which guarantee that the solutions have prescribed asymptotic behavior will be given.

In Chapter 4, we present a oscillation and nonoscillation theory for second order nonlinear differential equations of superlinear type. We begin with the oscillation of superlinear equations with sign changing coefficients. In particular, we discuss results which involve integrals and weighted integrals of the alternating coefficients, and then provide several criteria which use average behaviors of these integrals. More general averages such as 'weighted averages' and 'iterated averages' will also be employed. Also, we provide sufficient conditions which guarantee the existence of nonoscillatory solutions, and then we present necessary and sufficient conditions for oscillation of superlinear equations. Oscillation results via comparison of nonlinear equations of the same form as well as with linear ones of the same order will also be presented. We conclude this chapter by discussing oscillation and nonoscillation of forced and damped superlinear differential equations.

In Chapter 5, we present oscillation and nonoscillation criteria for all solutions of second order nonlinear differential equations of sublinear type with alternating coefficients. Our results will involve integrals and weighted integrals of alternating coefficients and in some results we use integral aver- 
aging techniques. We also provide some new linearized oscillation results for second order sublinear differential equations. Next, we study the EmdenFowler equations. These equations originated from the theory of gaseous dynamics in astrophysics around the beginning of the last century, and they also occur in the study of gas dynamics, fluid mechanics, relativistic mechanics, nuclear physics, and in chemically reacting systems. In this chapter we present criteria for nonoscillation of sublinear Emden-Fowler type equations. Also we compare the oscillatory behavior of certain nonlinear equations with related half-linear differential equations. We conclude this chapter by discussing oscillation of general nonlinear differential equations.

In Chapter 6 some special techniques are used to obtain oscillatory criteria for differential equations. Firstly, we present oscillation and nonoscillation theorems for nonlinear second order differential equations by using the method of Olech, Opial and Wazewski. Secondly, we use a variational inequality to study the oscillation of half-linear second order differential equations. Finally, we apply Liapunov second method to provide criteria for oscillation of second order nonlinear equations.

In Chapter 7, we discuss the oscillatory behavior of nonlinear twodimensional differential systems and second order vector-matrix differential equations. We begin with the oscillation of nonlinear two-dimensional differential systems. This includes the superlinear, linear, and sublinear cases. Then, we discuss oscillation of linear second order differential systems. Two approaches are presented. In the first approach the system considered will be reduced to a certain scalar Riccati inequality, so that known results from the literature can be applied. In the second approach, we will use Riccati and variational techniques. Next, we discuss the oscillation of nonlinear second order differential systems with functionally commutative matrix coefficients. We show that the oscillation theory of such systems can be effectively reduced to the study of diagonal systems of scalar second order differential equations. Then some comparison theorems of Hille-Wintner type for second order operator-valued linear differential equations will be presented. Finally, we discuss oscillation of second order differential systems with a forcing term.

The study of behavioral properties of solutions of differential equations near infinity is of immense importance in applications. In Chapter 8, we present some recent contributions on the asymptotic behavior of solutions of second order differential equations as well as the behavioral properties of positive solutions of singular Emden-Fowler-type equations. It is shown that for a large class of differential equations, not only can the existence of nonoscillatory solutions be proved, but also an explicit asymptotic form of 
the nonoscillatory solutions may be provided. Next, by imposing more restrictions on the sign of the integrable coefficient of the equation, we obtain necessary and sufficient conditions so that the solutions have a specified asymptotic behavior as $t \rightarrow \infty$, i.e., solutions which behave asymptotically like a nonzero constant and also those which behave asymptotically like $c t, c \neq 0$. For this, various averaging techniques of the type employed in the previous chapters to study the oscillatory behavior of such equations will be used. Then, we study existence, uniqueness and asymptotic behavior of positive solutions of singular Emden-Fowler-type equations. The cases when the coefficient of the equation under consideration is of constant sign, or of an alternating sign are systematically discussed. Finally, existence and nonexistence results for positive solutions of Emden-Fowler-type systems will be given.

In Chapter 9, some special results are presented. We extend the SturmPicone theorem, obtain nonoscillation theorems for perturbed second order nonlinear differential equations, and present a nonlinear Picone type identity which enables us to prove some Sturm-Picone type comparison theorems for nonlinear equations. Next, we study nonoscillatory solutions of forced differential equations of second order. Then, we present some limit cycle criteria and discuss its related properties for nonlinear second order differential equations. Finally, we provide some properties of solutions of very general second order differential equations.

In our final chapter, we introduce a nonoscillation theory for second order differential and neutral inclusions. Here, the results rely on some fixed point theorems for multivalued maps and on a compactness criterion.

We hope this book is timely and complements existing works in the literature on oscillation theory of second order dynamic equations. It can be used as a textbook at the graduate level and hopefully it will stimulate further research in this area.

It is impossible to acknowledge individually colleagues and friends to whom we are indebted for assistance, inspiration and criticism during the preparation of this book. We must, however, express our appreciation and thanks to Sadhna for typing and proof reading parts of the manuscript.

Ravi P Agarwal

Said R Grace

Donal O'Regan 